\title{
Products of all shapes and sizes for patients of all ages
}

At Curaprox, the team of specialists is dedicated to providing a range of oral health solutions suitable for patients of all ages.

Curaprox Baby created in collaboration with leading orthodontists is designed to ensure that babies get gentle yet effective oral care right from the very start. Products such as the bio functional soother size zero can be used from birth, while others, such as the award winning Easy Baby toothbrush, are ideal for small children using a toothbrush for the first time.

At the other end of the age scale is Curaprox's complete range of denture care solutions, which includes BDC 100 cleaning daily gel, BDC 105 weekly concentrate and specially designed denture brush with two sets of bristles for an efficacious clean.

For older children, adolescents and adults there is a wide mix of products available to suit every need, from manual and hydrosonic toothbrushes to interdental brushes, oral rinse and useful helpers such as tongue cleaners - there really is something for the whole family.

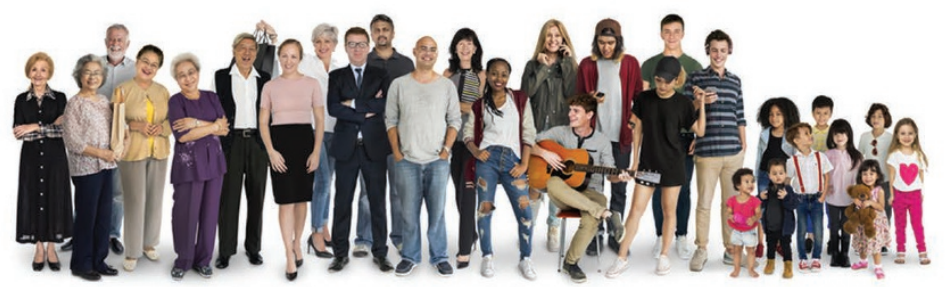

To supply Curaprox in your practice, call today.

For more information call 01480 862084, email info@curaprox.co.uk or visit www.curaprox.co.uk.

\section{How do you manage work-related stress?}

Dentistry has long been associated with stress but how you respond to it determines the level of impact it has on your work and wellbeing. What you need is a well-organised, streamlined system of support to call upon if you feel are under pressure or need assistance.

The London Smile Clinic is a dedicated referral centre that can take on complex cases and support you with expert help and advice. There is a team of highly qualified dentists offering services to your patients and along with their advanced clinical skills they have the time needed to focus on a five star experience and quality care.

The team ensures that referring dentists remain in control and are always kept fully informed until the patient is returned for continuing care. You can dispense with stress and rest assured that you can take on anything!

Contact the London Smile Clinic today on 02072552559 or visit www.londonsmile. co.uk/refer.

\section{Full mouth rehabilitation lecture this March}

There are still many patients who have failing, heavily restored dentitions that are not suitable for minimally invasive dentistry and require traditional full mouth rehabilitation.

This needs to be carried out at the highest level of precision in a structured, well planned manner using predictable and accurate techniques in order to ensure long term success. There needs to be close co-operation and understanding between the clinician and the technician as well as extremely close correlation between technical stages and clinical stages to avoid errors.

'Full mouth rehabilitation - sequencing and treatment', an all-day lecture by Mizrahi Dental Teaching, will be held on 23 March 2018 and will focus on the sequencing and treatment techniques needed. It will be aimed at experienced dentists and technicians and include how to assess and plan a full mouth rehabilitation; understand the steps involved and why each one is needed; demonstrate the techniques used including tooth preparation, core restorations, bite registration, temporisation and cementation; and troubleshooting.

Visit www.mizrahi-dental-teaching.co.uk or call 02033942984.
Reduce time, money and hassle

Nobel Biocare understands how important it is to optimise your time within the dental laboratory. Quicker turn around times mean happier patients and more satisfied clients, ultimately enhancing your reputation and increasing the chance of new referrals for business growth.

But how do you continue providing an outstanding service when you receive a sudden influx of implant bar prescriptions?

The Nobel Biocare Scan and Design Service will do the work for you so you can rest assured that your clients' needs will be met, even when you're already busy. Just send them the case, approve their design and receive the final implant bar ready to forward to the practice. You can even do the design stage yourself if you prefer - Nobel Biocare works around your needs.

Plus, you can have complete confidence in the precise fit of all bars provided thanks to accurate scan data, engineering, material and production expertise.

Whether you simply have too much work or you don't wish to invest in bar design software, production equipment or the relevant scan bodies, the Nobel Biocare Scan and Design Service provides a great alternative. Start saving time, money and hassle today.

For more information about Nobel Biocare, call 02087563300 or visit www.nobelbiocare.com.

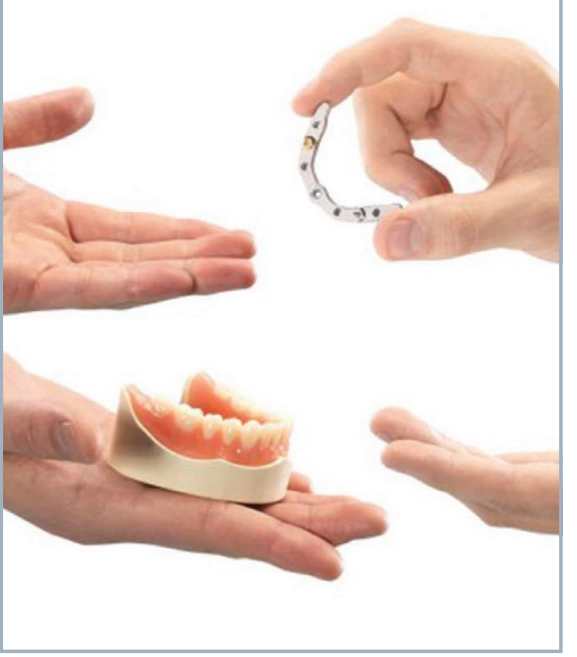

